\title{
PROJECTOR CALIBRATION USING A MARKERLESS PLANE
}

\author{
Jamil Draréni, Sébastien Roy \\ DIRO, Universite de Montreal , CP 6128 succ Centre-Ville, Montreal QC, Canada \\ drarenij@iro.umontreal.ca,roys@iro.umontreal.ca \\ Peter Sturm \\ INRIA Rhone-Alpes , 655 Avenue de lEurope, 38330 Montbonnot St Martin, France \\ Peter.Sturm@inrialpes.fr
}

Keywords: video projector calibration, planar calibration, focal estimation, structured light, photometric stereo

\begin{abstract}
In this paper we address the problem of geometric video projector calibration using a markerless planar surface (wall) and a partially calibrated camera. Instead of using control points to infer the camera-wall orientation, we find such relation by efficiently sampling the hemisphere of possible orientations. This process is so fast that even the focal of the camera can be estimated during the sampling process. Hence, physical grids and full knowledge of camera parameters are no longer necessary to calibrate a video projector.
\end{abstract}

\section{INTRODUCTION}

With the recent advances in projection display, video projectors (VP) are becoming the devices of choice for active reconstruction systems. Such systems like Structured Light (?) and Photometric Stereo (?; ?) use VP to alleviate the difficult task of establishing point correspondences. However, even if active systems can solve the matching problem, calibrated VP are still required. In fact, a calibrated projector is required to triangulate points in a camera-projector structured light system, or to estimate the projector's orientation when the latter is used as an illuminant device for a photometric stereo system.

Since a video projector is often modeled as an inverse camera, it is natural to calibrate it as part of a structured light system rather than as a stand alone device. In order to simplify the calibration process, a planar surface is often used as a projection surface on which features or codified patterns are projected. The projector can be calibrated as a regular camera, except for the fact that a regular accessory camera must be used to see the projector patterns. The way patterns are codified and the projection surface orientation is estimated will distinguish the various calibration methods from each other.

In (?), a VP projects patterns on a plane mounted on a mechanically controlled platform. Thus, the orientation and position of the projection plane is known and is used to calibrate the structured light system using conventional camera calibration techniques.

Other approaches use a calibrated camera and a planar calibration chessboard attached to the projection surface (?; ?).

For convenience and because the projection surface is usually planar, we will refer to it as the wall. The attached chessboard is used to infer the orientation and the position of the wall w.r.t the camera. This relation is then exploited, along with the images of the projected patterns to estimate the intrinsic parameters of the projector.

In order to measure the 3D position of the projected features, (?) estimates the homography between the attached chessboard and the camera. This allows the computation of the extrinsic parameters of the camera. It is important to mention that the camera must be fully calibrated in this case. With at least three different orientations, a set of 3D-2D correspondences can be obtained and then used to estimate the VP parameters with standard plane-based calibration methods (?; ?). We refer to this method as Direct Linear Calibration (DLC). To increase accuracy of the DLC, a printed planar target with circular markers is used in (?), to calibrate the camera as well as the projector.

In (?), a structured light system is calibrated without using a camera. This is made possible by embedding light sensors in the target surface. Gray- 
coded binary patterns are then projected to estimate the sensor locations and prewarp the image to accurately fit the physical features of the projection surface. The VP parameters are not explicitly estimated but the method could easily be extended for that purpose.

In this paper, a new projector calibration method is introduced. The proposed method does not require a physical calibration board nor a full knowledge of the camera parameters.

We overcome the problem of determining the camera-wall homography $\mathrm{H}_{w \rightarrow c}$ by exploring the space of all acceptable homographies and consider the one that minimizes the reprojection error (see Figure.1). Since $\mathrm{H}_{w \rightarrow c}$ depends only on the orientation between the camera and the wall, the space of acceptable homographies can be parameterized with only 2 angles : the elevation and the azimuth angles that define the normal vector at the wall.

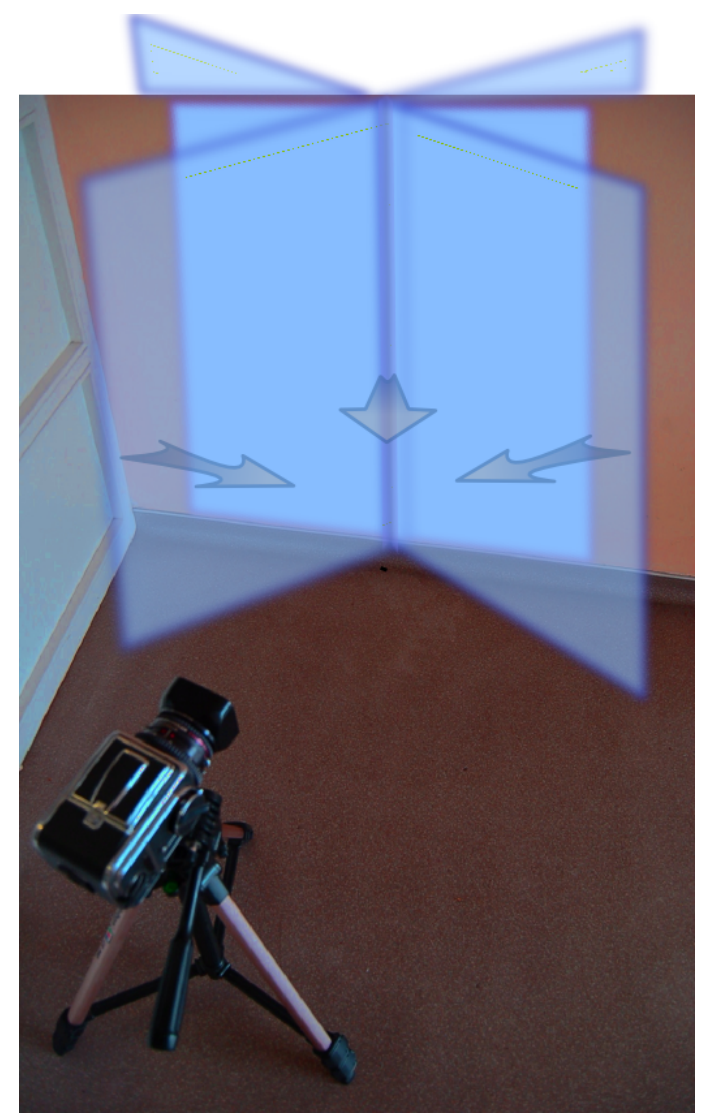

FIG. 1: The homography wall-camera is defined by the orientation of the wall.

Finding the normal of the wall consists then in sampling the space of orientations on a unit sphere. For each orientation sample, a DLC is performed and we select the homography that minimizes the reprojection errors in the images. It is worth mentioning that our DLC implementation differs slightly from the one used in (?) as explained in the next section.

Our proposed method is fully automatic, fast and produces excellent results as shown in our experiments. We also show that when the camera is not fully calibrated, projector calibration is still tractable. This is done by making the common assumptions that the pixels are square and that the center of projection coincides with the image center (?). Thus, the only unknown camera parameter left to estimate is the focal length, which is estimated by sampling.

The rest of this paper is organized as follows. Section 3 presents our variant of the direct linear calibration for a projector. Section 4 details our orientation sampling calibration (OSC) using only a (partially calibrated) camera and a marker-less projection plane.

Section 5 presents the results of our calibration method, followed by a discussion of limitations and future work in Section 6.

\section{Video Projector Model}

We model the video projector as an inverse camera. Therefore, we intend to compute the intrinsic and extrinsic parameters. Without loss of generality, we consider in this paper a 4 parameters projector model, namely : the focal length, the aspect ratio and the principal point. Thus, the projector matrix $\mathrm{K}_{p}$ is defined as :

$$
\mathrm{K}_{p}=\left(\begin{array}{ccc}
\rho f & 0 & c x \\
0 & f & c y \\
0 & 0 & 1
\end{array}\right)
$$

The extrinsic parameters that describe the $i^{\text {th }}$ projector pose are the usual rotation matrix $\mathrm{R}^{i}$ and the translation vector $\mathbf{t}^{i}$

\section{Direct Linear Calibration}

In this section, we review the details of the Direct Linear Calibration for projectors. This method is used as a reference for our benchmark test. As opposed to (?), the variant presented here is strictly based on homographies and does not require a calibrated camera.

If a static camera observes a planar surface (or a wall), a homography is induced between the latter and the camera image plane. This linear mapping $\left(\mathrm{H}_{w \rightarrow c}\right)$ relates a point $P_{w}$ on the wall to a point $P_{c}$ in the camera image as follows :

$$
\mathbf{P}_{c} \sim \mathrm{H}_{w \rightarrow c} \cdot \mathbf{P}_{w}
$$


Where $\sim$ denotes equality up to a scale. Details on homography estimation can be found in (?).

The video projector is used afterward to project patterns while it is moved to various positions and orientations. For a given projector pose $i$, correspondences are established between the camera and the VP, leading to a homography $\mathrm{H}_{c \rightarrow p}^{i}$. A point $\mathbf{P}_{c}^{i}$ in the image $i$ is mapped into the projector as :

$$
\mathbf{P}_{p}^{i} \sim \mathrm{H}_{c \rightarrow p} \cdot \mathbf{P}_{c}^{i}
$$

Combining Eq. 1 an Eq.2, a point $\mathbf{P}_{w}$ on the wall is mapped into the $i^{\text {th }}$ projector as :

$$
\mathbf{P}_{p}^{i} \sim \underbrace{\mathrm{H}_{c \rightarrow p}^{i} \cdot \mathrm{H}_{w \rightarrow c}}_{\mathrm{H}_{w \rightarrow p}^{i}} \cdot \mathbf{P}_{w}
$$

On the other hand, $\mathbf{P}_{p}^{i}$ and $\mathbf{P}_{w}$ are related through a perspective projection as :

$$
\mathbf{P}_{p}^{i} \sim \mathrm{K}_{p} \cdot\left[\mathbf{R}_{1}^{i} \mathbf{R}_{2}^{i} \mathbf{t}^{i}\right] \cdot \mathbf{P}_{w}
$$

Where $\mathrm{K}_{p}, \mathbf{R}_{1,2}^{i}$ and $\mathbf{t}^{i}$ are respectively the projector intrinsic parameters, the two first vectors of the rotation matrix $\mathrm{R}^{i}$, and the translation vector. From Eq.3 and Eq.4, a relation between $\mathrm{H}_{w \rightarrow p}^{i}$ and the extrinsic parameters of the projector is derived as follows :

$$
K_{p}^{-1} \cdot \mathrm{H}_{w \rightarrow p}^{i} \sim\left[\mathbf{R}_{1}^{i} \mathbf{R}_{2}^{i} \mathbf{t}^{i}\right]
$$

With at least two different orientations, one can solve for $\mathrm{K}_{p}^{-1}$ by exploiting the orthonormal property of the rotation matrix as explained in (?).

\section{Orientation Sampling Calibration}

In this section we give the details of our proposed video projector calibration method. As discussed earlier, the justification for using an attached calibration rig to the wall is to infer the homography wall-camera in order to estimate the $3 \mathrm{D}$ coordinates of the projected features. We propose to estimate this wall-camera relation by exploring the space of all possible orientations since only the orientation of the wall w.r.t the camera matters and not its position.

Another way to look at this orientation space is to consider all vectors lying on a unit hemisphere placed on the wall, as depicted on Figure 1.

The calibration process can be outlined in three main steps :

- Pick a direction on the hemisphere.

- Compute the corresponding homography.

- Use the homography to perform a DLC calibration (Section 3).
The above steps are repeated for all possible directions and the direction that minimizes the reprojection errors is selected as the correct plane orientation. The first two steps are detailed in the next subsections. The third one is straightforward from section 3 .

\subsection{Sampling a Hemisphere}

The problem of exploring the set of possible orientations is dependent on the problem of generating uniformly distributed samples on the unit sphere (hemisphere in our case).

Uniform sphere sampling strategies can be random or deterministic (?). The first class are based on random parameters generation, followed by an acceptance/rejection step depending on whether the sample is or not on the sphere. Deterministic methods produce valid samples on a unit sphere from uniformly distributed parameters, such method include (but not limited to) quaternion sampling (?), normal-deviate methods (?) and methods based on Archimedes theorem (?). We chose to use the latter method for its simplicity and efficiency. As the name suggests, this method is based on Archimedes theorem on the sphere and cylinder which states that the area of a sphere equals the area of every right circular cylinder circumscribed about the sphere excluding the bases. This argument leads naturally to a simple sphere sampling algorithm based on cylinder sampling (?). Uniformly sampling a cylinder can be done by uniformly choosing an orientation $\theta_{i} \in[0, \pi]$ (we call it azimuth) to obtain a directed vector $d\left(\theta_{i}, 0\right)$ (See Figure.2). After that, a height $h_{i}$ is uniformly chosen in the range $[-1,1]$. The resulting vector, noted $d_{i}\left(\theta_{i}, h_{i}\right)$, is axially projected on the unit sphere. According to the above theorem, if a point is uniformly chosen on a cylinder, its inverse axial projection will be uniformly distributed on the sphere as well, see (?) for further details.

In our case, we only need to sample the hemisphere facing the camera. Thus the span of the points that must be visited is limited to the range $[-1,+1] \times$ $[0, \pi]$.

\subsection{Homography From an Orientation Sample}

The homography wall-camera $\mathrm{H}_{w \rightarrow c}^{i}$ induced by a wall whose normal is a direction $\mathbf{d}_{i}$ (as defined in the previous subsection), is defined by :

$$
\mathrm{H}_{w \rightarrow c}^{i} \sim \mathrm{K}_{\text {cam }} \cdot\left[\mathbf{R}_{1}^{i} \mathbf{R}_{2}^{i} \mathbf{t}\right]
$$

Where $\mathrm{K}_{\text {cam }}, \mathbf{R}_{1}^{i}, \mathbf{R}_{2}^{i}$ and $\mathbf{t}$ are respectively the intrinsic camera matrix, the first two vectors of the ro- 


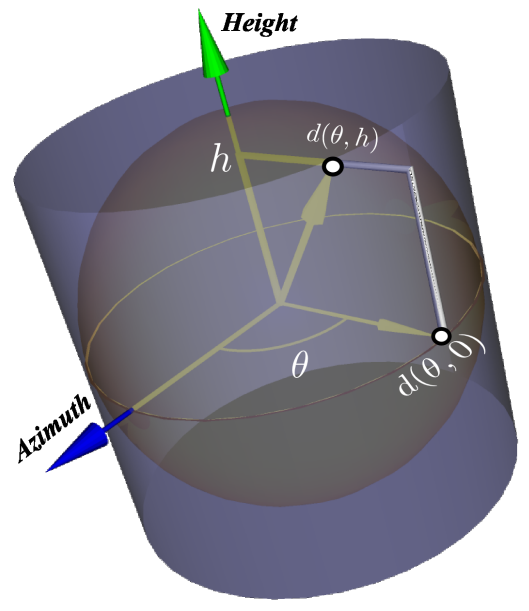

FIG. 2: Orientation space sampling.

tation corresponding to the direction $\mathbf{d}_{i}$, and the translation vector. Without loss of generality and for the sake of simplicity, we fix the projection of the origin of the wall $P_{w}^{0}=(0,0)^{\top}$ into the camera at the image center. With this convention, the translation vector $\mathbf{t}$ simplifies to $(0,0,1)^{\top}$.

The rotation matrix $\mathrm{R}^{i}$ is computed via Rodrigues formula, which requires a rotation axis and a rotation angle. The rotation axis is simply the result of the cross product between $\mathbf{d}_{i}$ and the vector $(0,0,1)^{\top}$ whereas the rotation angle $\alpha_{i}$ is obtained from the dot product of the same vectors :

$$
\alpha_{i}=\cos ^{-1}\left(\mathbf{d}_{i}^{\top} \cdot(0,0,1)^{\top}\right)
$$

\subsection{Complete Algorithm}

We are now ready to give the complete algorithm of our video projector calibration. We assume the existence of two supporting functions, ReprojError that returns a reprojection error for a given projector parameters and $D L C$ a function that estimate the projector parameters using the DLC method (see Section.3).

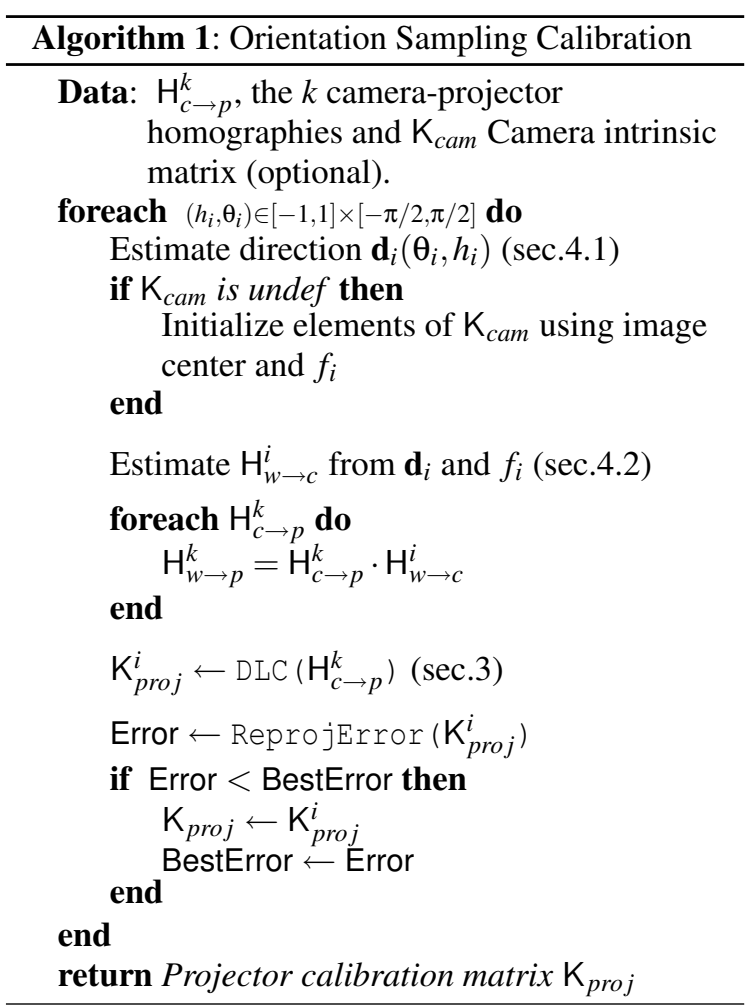

\section{EXPERIMENTS}

We have evaluated the proposed calibration method with both a calibrated and an uncalibrated cameras. The results were also compared to the DLC method. The evaluation platform consists of a Mitsubishi pocket projector of $800 \times 600$ pixels resolution and a digital camera (Nikon D50). A $50 \mathrm{~mm}$ lens was used on the camera and the resolution was set to $1500 \times 1000$. The calibration of the camera using the Matlab toolbox gave the following intrinsic matrix $\mathrm{K}_{\text {cam }}$ :

$$
\mathrm{K}_{\text {cam }}=\left(\begin{array}{ccc}
3176.3115 & 0 & 790.6186 \\
0 & 3172.4809 & 495.3829 \\
0 & 0 & 1
\end{array}\right)
$$

To include the DLC algorithm in our benchmark, the camera was mounted on a tripod and was first registered to the wall using an attached printed chessboard. Images of projected chessboard using the video projector under several orientations were then acquired using the camera. We took precaution to remove the attached chessboard form the wall before acquiring the projector images to avoid overlaps between the projected patterns and the rigidly attached pattern. 
Some images of the projected chessboard along with detected features are depicted on Figure.3.

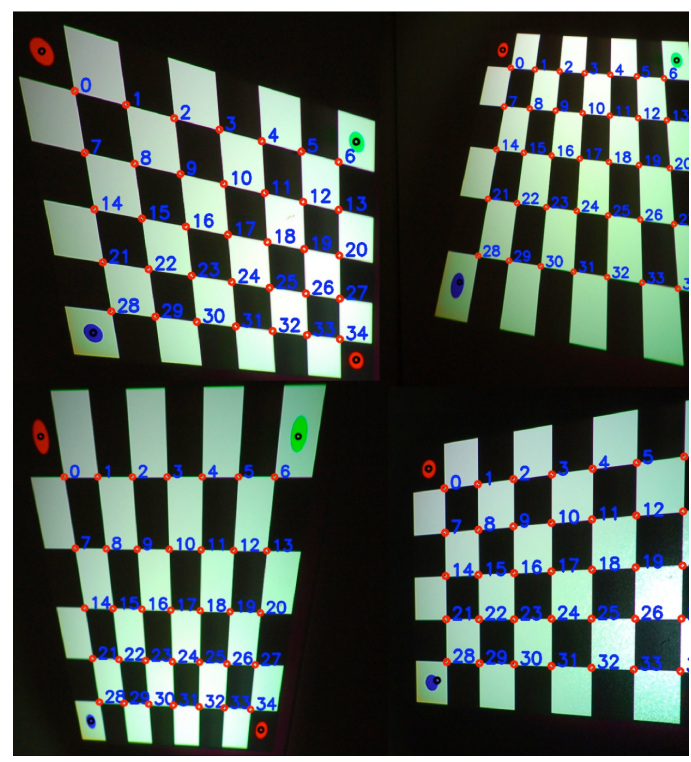

FIG. 3: Images of projected patterns and detected features. The numbers and small red dots are added for illustration only. The large dots in the 4 corners are part of the projected pattern.

Notice the presence of colored dots on the chessboard. Those were used to compute a rough estimate of the homography (which will be refined) and to eliminate the orientation ambiguity of the chessboard while assigning $3 \mathrm{D}$ coordinates to the detected features.

Our benchmark includes a projector calibration using the DLC method, the proposed method with both a calibrated and an uncalibrated camera. In the first case, we used the image of the attached checker to infer the wall-camera homography and calibrated as explained in Section.3. For the second method, we used a multi-resolution strategy to sample the azimuth angles and heights. The conditions of the third method were identical to the second one except that the camera parameters were ignored and were estimated as follows :

- The focal length estimation was included in the sampling process. The sampling range was $[0,10000]$.

- The pixels are assumed square.

- The center of projection is assumed to coincides with the image center.

TAB. 1: Projector calibration benchmark : Direct method, Orientation sampling with a calibrated camera (SamplingC) and Orientation sampling with an uncalibrated camera (Sampling-U).

\begin{tabular}{|c||c|c|c|c|c|c|c|}
\hline Method & $\mathbf{f}_{\text {proj }}$ & $\rho$ & $\mathbf{c x}$ & cy & estf $_{\text {cam }}$ & Error & Error B.A \\
\hline \hline Direct & 1320.13 & 1.02 & 382.1 & 368 & - & 4.35 & 0.47 \\
Sampling-C & 1327.30 & 1.01 & 377.4 & 366 & - & 0.43 & 0.22 \\
Sampling-U & 1322.15 & 1.00 & 376 & 360 & 3108 & 0.16 & 0.09 \\
\hline
\end{tabular}

The result of this benchmark is outlined on the Table.1. The table provides the estimated parameters, the reprojection errors in pixels (Error), and the error difference comparing before and after applying a bundle adjustment refinement (Error B.A). Technical and implementation details on the latter can be found in (?).

The running times for a data set of 20 images on an $1.5 \mathrm{Ghz}$ computer are provided in Table.2.

TAB. 2: Execution time for Direct method, Sampling with calibrated camera, and Sampling with uncalibrated camera.

\begin{tabular}{|c||c|}
\hline Method & Time (seconds) \\
\hline Direct & 0.18 \\
Sampling-C & 1.23 \\
Sampling-U & 6.2 \\
\hline
\end{tabular}

From this test, we can see that our method, even in the absence of camera parameters knowledge, outperform the Direct Linear Method at the expenses of a higher running time. However, we are convinced that the performance of our implementation could be further improved by choosing a better multi-scale sampling strategy. We also consider that not requiring a printed chessboard attached to the wall is a major advantage, especially when the wall surface is large or unaccessible.

A plot of the reprojection error in terms of the orientation parameters $h$ and $\alpha$ is provided in Figure.4. We can clearly see that the function is very well behaved and easy to minimize.

As a last test, we wanted to assess the stability of the focal length estimate. We thus fixed the value of the wall orientation at the value obtained in the first experiment and varied the focal length. The plot of the reprojection error as a function of the sampled focal length of the camera is shown on Figure.5. As we can see the error function is smooth and convex, suggesting that the lack of knowledge of the focal length can easily be circumvented in practice.

\section{CONCLUSION}

In this paper we presented a new video projector calibration method. Contrary to most methods, we sho- 


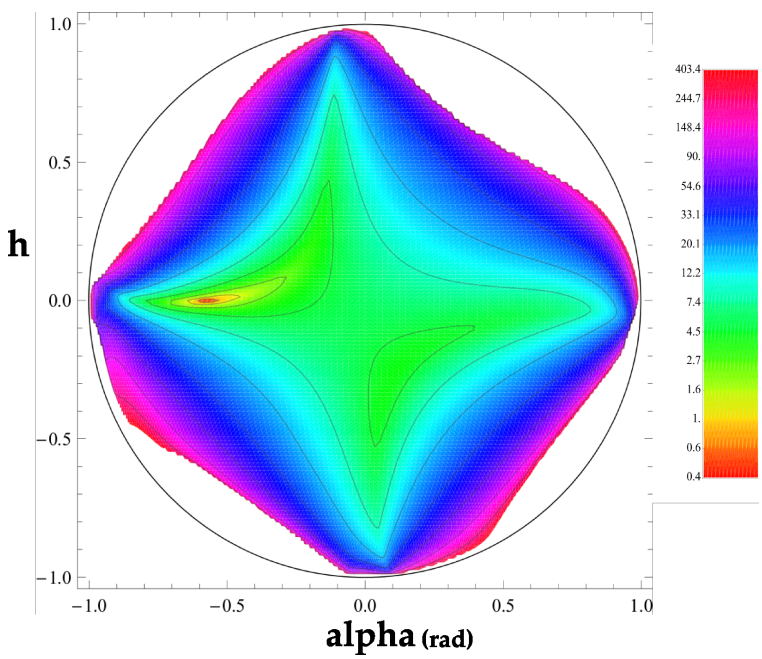

FIG. 4: Reprojection error in terms of the orientation parameters $h$ and $\alpha$. The error computation does not include bundle adjustment refinement

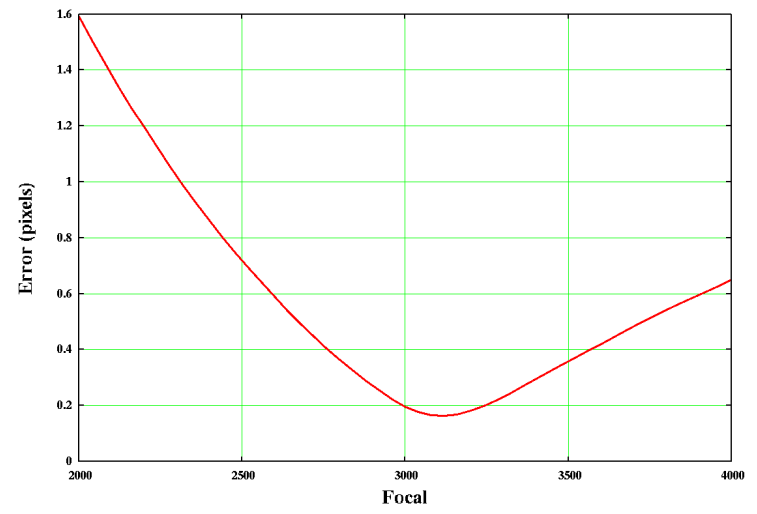

FIG. 5: Reprojection error in terms of the camera focal length values (prior to bundle adjustment procedure). The minimum is reached at 3034.4 , the off-line camera calibration estimated a camera focal of 3176 .

wed that a physical target attached to a projection surface is not necessary to achieve an accurate projector calibration. We also suggest that full knowledge of camera parameters is not strictly required and can be relaxed into a set of commonly used assumptions regarding the camera geometry. Very simple to implement, the proposed method is fast and will handle large projector-camera systems that were previously impossible to calibrate due to the impractical chessboard.

\section{REFERENCES}

Barsky, S. and Petrou, M. (2003). The 4-source photometric stereo technique for three-dimensional surfaces in the presence of highlights and shadows. IEEE Transactions on Pattern Analysis and Machine Intelligence, 25(10) :1239-1252.

Hartley, R. I. and Zisserman, A. (2004). Multiple View Geometry in Computer Vision. Cambridge University Press, ISBN : 0521540518, second edition.

Horn, B. K. P. (1986). Robot Vision (MIT Electrical Engineering and Computer Science). The MIT Press, mit press ed edition.

Knuth, D. E. (1997). Art of Computer Programming, Volume 2 : Seminumerical Algorithms (3rd Edition). Addison-Wesley Professional.

Lee, J. C., Dietz, P. H., Maynes-Aminzade, D., Raskar, R., and Hudson, S. E. (2004). Automatic projector calibration with embedded light sensors. In Proceedings of the 17th annual ACM symposium on User interface software and technology, pages 123-126. ACM.

Lourakis, M. and Argyros, A. (2004). The design and implementation of a generic sparse bundle adjustment software package based on the levenberg-marquardt algorithm. Technical Report 340, Institute of Computer Science FORTH, Heraklion, Crete, Greece. Available from http://www.ics.forth.gr/ lourakis/sba.

Min-Zhi Shao, N. B. (1996). Spherical sampling by archimedes' theorem. Technical Report 184, University of Pennsylvania.

Ouellet, J.-N., Rochette, F., and Hébert, P. (2008). Geometric calibration of a structured light system using circular control points. In 3D Data Processing, Visualization and Transmission, pages 183-190.

Sadlo, F., Weyrich, T., Peikert, R., and Gross, M. (2005). A practical structured light acquisition system for pointbased geometry and texture. In Proceedings of the Eurographics Symposium on Point-Based Graphics, pages 89-98.

Salvi, J., Pagés, J., and Batlle, J. (2004). Pattern codification strategies in structured light systems. Pattern Recognition, 37(4) :827-849.

Shen, T. and Meng, C. (2002). Digital projector calibration for 3-d active vision systems. Journal of Manufacturing Science and Engineering, 124(1):126-134.

Snavely, N., Seitz, S. M., and Szeliski, R. (2006). Photo tourism : Exploring photo collections in $3 \mathrm{~d}$. In SIGGRAPH Conference Proceedings, pages 835-846, New York, NY, USA. ACM Press.

Sturm, P. and Maybank, S. (1999). On plane-based camera calibration : A general algorithm, singularities, applications. In Proceedings of the IEEE Conference on Computer Vision and Pattern Recognition, Fort Collins, USA, pages 432-437.

Woodham, R. J. (1978). Photometric Stereo : A Reflectance Map Technique for Determining Surface Orientation from a Single View. In Proceedings of the $22^{\text {nd }}$ SPIE Annual Technical Symposium, volume 155, pages 136-143, San Diego, California, USA. 
Yershova, A. and LaValle, S. M. (2004). Deterministic sampling methods for spheres and so(3). In ICRA, pages 3974-3980.

Zhang, Z. (1999). Flexible camera calibration by viewing a plane from unknown orientations. Computer Vision, 1999. The Proceedings of the Seventh IEEE International Conference on, 1 :666-673 vol.1. 